\title{
A NEW METHOD FOR DETERMINING THE LONG-PERIOD COMPONENT OF THE SOURCE TIME FUNCTION OF LARGE EARTHQUAKES
}

\author{
Jiajun Zhang and Thome Lay
}

Department of Geological Sciences, The University of Michigan

\begin{abstract}
Inversions of seismic body waves indicate that most large earthquakes involve spatial and temporal heterogeneity of the moment release, but seismic body waves usually lack sufficient bandwidth to constrain the long-period component of the radiation. Analysis of long-period surface waves and free oscillations can constrain the overall source duration and moment; however, most procedures assume simple trapezoidal or boxcar source-time histories, inconsistent with the body wave complexity. We find that source-time function complexity can affect long-period surface waves sufficiently to impact estimates of the source moment tensor, rupture duration and centroid depth. We present a procedure to objectively determine the long-period component of complex source-time functions in which we directly utilize results from body wave analysis. The method is applied to two great normal fault events of June 22, 1977 (Tonga, $M_{W}=8.2$ ) and August 19, 1977 (Indonesia, $M_{W}=8.5$ ). Standard long period analysis procedures underestimate the total slip duration in both cases. The overall source process times of both earthquakes are longer than $120 \mathrm{~s}$.
\end{abstract}

\section{Introduction}

Investigation of large earthquake ruptures provides important information on the physical processes occurring at plate boundaries. Detailed seismic body wave inversions indicate that most events consist of several subevents which represent temporal and spatial inhomogeneities of the moment release during the rupture process [e.g., Schwartz and Ruff, 1985; Hartzell and Heaton, 1985; Kikuchi and Fukao, 1987]. The resulting complex source time functions are often interpreted as reflecting variation in slip on the fault, or fluctuations in areal expansion rate of the rupture front. Since the signal wavelengths used in body wave analysis are relatively short, the details of fault rupture or source complexity can be well determined. However, for very large earthquakes with moment magnitudes greater than about $M_{W}=8.0$, the body waves usually lack sufficient bandwidth to resolve the total source duration and moment release so there is significant ambiguity in inferring the slip distribution. To determine the longperiod component or overall characteristics of the source process of large earthquakes, long-period seismic waves (with periods greater than $100 \mathrm{~s}$ ) must be used. Since long-period surface waves and free oscillations provide better resolution of the overall faulting process, the moments and source durations from such analyses are usually combined with the body wave results to interpret the source process. However, this is seldom done in a self-consistent fashion.

Our insufficient knowledge of propagation effects on shortperiod surface waves prevents complete resolution of earthquake source process using surface wave data alone. As a result, most long period analyses assume simple moment-rate function parameterizations in the form of boxcars (i.e. ramp dislocation functions) or trapezoids (i.e. Haskell source models), regardless

Copyright 1989 by the American Geophysical Union.

Paper number $89 \mathrm{GL} 00377$.

$0094-8276 / 89 / 89 \mathrm{GL}-00377 \$ 03.00$ of any complexities required by the body wave radiation [e.g., Kanamori and Given, 1981; Dziewonski and Woodhouse, 1983]. This is valid for small events with rupture durations much shorter than the periods of the waves being analyzed, but for great events this assumption must be appraised. Yet only a few procedures have been introduced to analyze the body wave and surface wave data jointly [e.g., Ekström and Dziewonski, 1986], and typically the results from the different wave types are combined in an ad hoc fashion by smoothly adding the missing long-period moment to the body wave source function over a specified duration [Kikuchi and Fukao, 1987; Beck and Ruff, 1987].

The determination of source depth from seismic waves requires an accurate description of the source-time function. The tradeoffs between depth and time function are severe when using waves with a narrow bandwidth. In this paper we will present a procedure for incorporating the results of body wave analysis of moment-rate function complexity into the inversion of longperiod surface waves for the overall source process. The introduction of the source complexity into the inversion improves the accuracy of the source-finiteness correction to the long-period complex spectra, which is critical for the determination of the low-frequency characteristics of the seismic source. By application of this method to two great earthquakes we will show that not only do we obtain an internally consistent broadband source function, but we also find significantly different values of source depth, total moment and source duration compared with standard inversions.

\section{Procedure}

We use the basic moment tensor inversion approach developed for surface waves by Kanamori and Given [1981] as modified to a two-step inversion algorithm by Zhang and Kanamori [1988a,b (hereinafter referred to as $\mathrm{ZKa}$ and $\mathrm{ZKb}$, respectively)]. The data are the digital seismograms recorded by the Global Digital Seismograph Network (GDSN) and International Deployment of Accelerometers (IDA) networks. The complex spectra of vertical component seismograms of Rayleigh waves of periods from 150 to $300 \mathrm{~s}$ are used. Propagation corrections for travel times are made using the laterally heterogeneous earth model (M84C) obtained by Woodhouse and Dziewonski [1984], and the amplitudes are corrected for the $\mathrm{Q}$ model obtained by Dziewonski and Steim [1982]. First, we invert the spectra of a given period by solving the following system for a step-function point source of a moment tensor [ZKb]:

$$
B D=V
$$

where $D$ is the solution vector involving products of the moment tensor elements and corresponding excitation functions, and $\mathrm{V}$ the data vector calculated from the observed spectra vector by correcting for the effects of propagation, instrument response, and an assumed source finiteness model. Equation (1) is solved by least squares, and the weighted RMS error, which measures the misfit between the data and the given source finiteness model, is given by $\sigma(\omega)=\varepsilon(\omega)\left[\Sigma\left|\hat{R}_{i}(\omega)\right|^{2} / N\right]^{1 / 2}$, where $\varepsilon(\omega)$ is the RMS error in solving $(1), \hat{R}_{i}(\omega)$ is the effect of the finiteness due to the given model, and $N$ is the number of records used in the inversion. Since the RMS error depends on the source finiteness model used to correct the observed spectra, the first step is to isolate the source finiteness effect. 
The second step is to search for the best depth and determine the moment tensor by solving the following system [ZKb]:

$$
\Gamma M=\Lambda
$$

where $\Gamma$ is a matrix of excitation coefficients for a given source depth and earth model, $M$ is the solution vector representing a moment tensor, and vector $\Lambda$ is a stack of vector D's obtained by solving (1) at several periods using a given model of the moment-rate function. $\Lambda$ has $5 K$ elements; $K$ is the number of periods. We solve (2) by least squares and find the depth that minimizes the non-dimensional error: $p=\left[p_{i} p_{i}\right]^{1 / 2}$, where the summation convention is used and $\rho_{i}=\left(\Lambda_{i}-\hat{\Lambda}_{1}\right) /\left(\Lambda^{T} \Lambda\right)^{1 / 2}$ $(i=1, \ldots, n)$ with $\mathrm{n}=5 \mathrm{~K}$. Here $\Lambda_{1}$ and $\hat{\Lambda}_{i}$ are the observed and predicted components of vector $\Lambda$ in (2). Ideally, we would find minima for $\rho$ and $\sigma$ for identical complex source parameter combinations; however, limitations intrinsic to long-period surface waves require that constraints from body waves be used in the analysis.

The temporal variation of the source is usually represented by a point source with a synchronous moment-rate tensor $\mathbf{M}(t)$ :

$$
M_{i j}=f(t) \sqrt{2} \hat{M}_{i j}, \quad(i, j=1,2,3)
$$

(in Cartesian tensor notation everywhere in this paper), where $f(t)$ is the moment-rate function, $\hat{\mathbf{M}}$ is a real constant tensor of unit Euclidean norm $\left(\hat{M}_{i j} \hat{M}_{i j}=1\right)$. For an arbitrary moment tensor source that varies as a step function in time, $f(t)$ is a delta function. Using the notation of Silver and Jordan [1982], $\mathbf{M}(\omega)=\sqrt{2} M_{T}(\omega) \hat{\mathbf{M}}(\omega)$, it follows that their total moment, $M_{T}(\omega)=|f(\omega)|$, and unit tensor, $\hat{\mathbf{M}}(\omega)=\hat{\mathbf{M}} \exp [i \phi(\omega)](f(\omega)$ $=|f(\omega)| \exp [i \phi(\omega)]$ denotes the Fourier transform of $f(t))$.

Usually, the source finiteness corrections are made assuming a boxcar or trapezoid for the moment-rate function. We will adopt a more general parameterization so that the source model is consistent with body waves. In this parameterization, the complexity in the shape of the moment-rate function is represented using a composite of half-sine functions: $\Pi_{i}(t)=(\pi / 2) \Pi_{0}(t) \sin \left[\pi\left(t-t_{t}\right) / \tau_{t}\right]$, where $t_{i}$ and $\tau_{i}$ are subevent start time and process time, and $\Pi_{0}(t)=\left[H\left(t-t_{i}\right)-H\left(t-t_{i}-\tau_{i}\right)\right] / \tau_{i}$ is a boxcar function. Using a series of these half-sine functions, each with its own start time, $t_{i}$, and process time, $\tau_{i}$, we construct various models of moment-rate functions, which allow for a moment-rate function consistent with body wave radiation to be included. Typically, the parameters to be determined are the relative moment and process time of the long-period component of the source process. The total process time, $\tau$, which represents the entire interval of source radiation, is distinct from the apparent source duration which we define as

$$
t_{s}=\frac{1}{f_{\max }} \int_{l_{i}}^{t_{i}+\tau} f(t) d t
$$

where $f(t)$ has the height $f_{\max }$ and is time-limited within the interval of integration defined by the start time $t_{i}$ and $\tau$. $t_{s}$ is the base of a rectangle with height $f_{\max }$ and area equal to the area of the $f(t)$ curve, thus it is always less than $\tau$ except for a boxcar function.

For simplicity, we call the integral in (4) the seismic moment $M_{0}: M_{0}=f_{\max } t_{s}$, which is the static moment or zero-frequency total moment, $M_{T}^{o} \equiv M_{T}(0)$, of Silver and Jordan [1982]. If $\hat{\mathbf{M}}$ is deviatoric, there exists $M_{O M D C} \geq M_{0} \geq M_{O B D C}$, where $M_{O M D C}$ and $M_{0 B D C}$ are the seismic moments of the major double couple [Gilbert, 1980] and best double couple [Dziewonski and Woodhouse, 1983], respectively. If $\hat{\mathbf{M}}$ is almost a double couple, then $M_{O B D C}$ is much closer to $M_{0}$ than $M_{\text {OMDC }}\left(M_{0}-M_{0 B D C} \ll M_{O M D C}-M_{0}\right)$. The solution vector in (2), $M$, represents the moment tensor $M_{0} \sqrt{2} \hat{M}_{i j}$.

The centroid and variance of $f(t)$ are often used to measure the finiteness of the source process. The centroid time is given by $t_{c}=1 / M_{0} \int_{i}^{t_{i}+\tau} f(t) t d t$, and the variance time or characteristic time [Silver and Jordan, 1983] is given by $\tau_{c}=\left[4 / M_{0} \int_{t_{\mathrm{i}}^{i}}^{t_{i}+\tau_{f}} f(t)\right.$ $\left.\left(t-t_{c}\right)^{2} d t\right]^{1 / 2} . t_{s}, t_{c}$, and $\tau_{c}$ correspond to polynomial moments of order 0,1 and 2 of $f(t)$, respectively. For a Haskell source, which is a point source characterized by a rise time $t_{R}$ and rupture time $t_{f}, f(t)$ is a trapezoid with $t_{s}=t_{R}+t_{f}, \tau=2 t_{R}+t_{f}$, $t_{c}=t_{R}+t_{f} / 2$, and $\tau_{c}=\left[\left(t_{R}^{2}+t_{f}^{2}\right) / 3\right]^{1 / 2}$.

\section{Application}

\section{June 22, 1977 Tonga earthquake}

For the Tonga earthquake (1208:33.4UT, $22.88^{\circ} \mathrm{S}, 175.90^{\circ} \mathrm{W}$, $m_{b}=6.8, M_{s}=7.2, M_{W}=8.2$ ), body wave studies indicate that the high frequency source process consists of two major subevents spanning a combined process time of $50 \mathrm{~s}$ after the origin time [Christensen and Lay, 1988]. We model the Tonga source function as a composite of three subevents given by halfsine pulses $\Pi_{i}$ : two pulses approximating the body wave time function as well as a long duration component representing any additional radiation.

We determine the process time and relative moment of the third, long-period component of the Tonga source process using the variable moment-rate functions $(\mathrm{V}-\mathrm{m})$ in the inversion procedure. Figure 1a shows our preferred model, which comprises three components with a total process time of $165 \mathrm{~s}$. The moment-rate function is obtained by assuming that the first and second components, starting at 0 and $25 \mathrm{~s}$ respectively, have the same process time, $25 \mathrm{~s}$, and the same moment $\left(m_{1}=m_{2}\right)$, as indicated by body waves [Christensen and Lay, 1988]; with the third component, starting at $0 \mathrm{~s}$, having a moment $\left(m_{3}\right) 40 \%$ of the total moment, $m_{1}+m_{2}+m_{3}$. The relative moment assigned to the third component is estimated by comparing errors, $p$ and $\sigma$, obtained for various assumed moment ratios, $m_{3} /\left(m_{1}+m_{2}+\right.$ $m_{3}$ ).

Figures $1 b$ and $1 c$ show $\rho, \sigma$, process time, and seismic moment for the Tonga event obtained for various moment ratios. Here $\sigma$ is shown for a 256-s period, which we consider more reliable than other periods. For a moment ratio of one, which corresponds to a single half-sine moment-rate function, $\sigma, \rho$, and process time are about the same as obtained assuming a boxcar moment-rate function. For a ratio of about $40 \%, \rho$ is minimized; the process time is $165 \mathrm{~s}$; and the seismic moment is the maximum, $21.5 \times 10^{20} \mathrm{~N} \mathrm{~m}$. For a small ratio (less than $40 \%$ ) which corresponds to a moment-rate function with a small third component, there is no process time with sufficient phase delay to satisfy the data; so $\sigma$ is large. The process times and seismic moments shown in Figure 1c for moment ratios less than 0.4 are for the moment rate which has the maximum phase delay. We
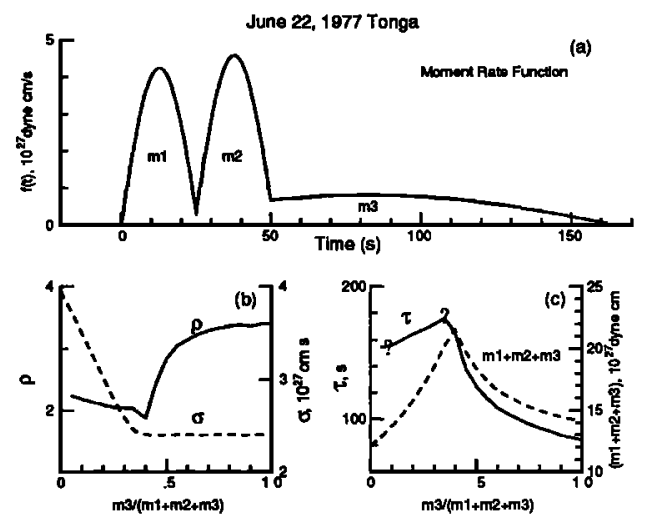

Fig. 1. The Tonga earthquake results. (a) The moment-rate function. (b) The errors, $\rho$ (solid line) and $\sigma$ (256-s period) (dashed line), versus moment ratio curves. (c) The process time, $\tau$, (solid line) and total seismic moment, $m_{1}+m_{2}+m_{3}$, (dashed line) versus moment ratio curves. 
conclude that the moment-rate functions with a small ratio are not acceptable. Our preferred total moment estimate of $21.5 \times$ $10^{20} \mathrm{~N} \mathrm{~m}$ and the average body wave moment of $12-15 \times 10^{20} \mathrm{~N}$ $\mathrm{m}$ [Christensen and Lay, 1988] indicate that about 30-45\% of the moment is missed by the body waves. This is consistent with our moment rate parameterization, which assigns $40 \%$ of the total moment to the very long period component.

Accounting for the realistic shape of the time function of the Tonga earthquake improves the long-period estimates of the seismic moment and source mechanism compared to standard assumptions of a boxcar or trapezoid time function. The source finiteness model shown in Figure 1a gives a better fit to the Rayleigh wave data than any of the other source finiteness models tested, as indicated by the minimum value of $\rho$ for this model. Although the details of the source finiteness model cannot be determined uniquely, the use of the source time function determined from body waves to constrain part of the source finiteness model improves the moment tensor solution from the long-period surface waves in this case.

Figure 2a shows the $p$ versus depth curves for two momentrate functions and two earth models: the average ocean model of Regan and Anderson [1984] (hereinafter referred to as R-A) and PREM [Dziewonski and Anderson, 1981]. We consider the model R-A to be more appropriate for earthquakes in the Tonga region. For the constant moment-rate function $(\mathrm{C}-\mathrm{m})$, i.e. a boxcar, with an optimal 84-s process time (or duration) determined using the method of $\mathrm{ZKa}$, the centroid depth is $91 \mathrm{~km}$ for R-A and $98 \mathrm{~km}$ for PREM, respectively. For the more realistic $V-m$ model with an 165-s process time, the centroid depth is $96 \mathrm{~km}$ with a $(93,104) 90 \%$ confidence depth range for R-A and 103 $\mathrm{km}$ with a $(99,112) 90 \%$ confidence depth range for PREM, respectively, found using a Student's $t$ test [ZKb]. The errors in the inversion using the variable moment-rate function have a lower mean (about $45 \%$ smaller) and smaller variance with $90 \%$ confidence (using the Siegel-Tukey test) for both earth models compared to the errors for the constant moment-rate function. Moreover, the variable moment-rate function fits the body wave radiation, while the constant moment-rate function does not. The moment tensor solution obtained by using $\mathrm{R}-\mathrm{A}$ for a point source at $96-\mathrm{km}$ depth with the variable moment-rate function is our preferred solution for the Tonga earthquake.

This estimate of the centroid depth is somewhat greater than the $65-\mathrm{km}$ depth of the earthquake hypocenter determined from the first $P$ wave arrivals (NEIS). This indicates that much of the long-period energy of the earthquake is released at depths below the depth of initiation of the earthquake. The confidence bounds on depth are obtained for fixed models of source finiteness, exci-

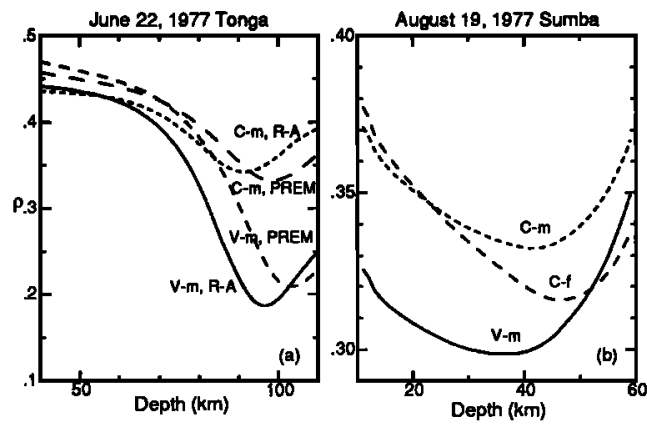

Fig. 2. (a) Error $\rho$ in the inversion for the Tonga event obtained for two source time functions: the constant (boxcar) moment rate function $(\mathrm{C}-\mathrm{m})$, and the variable source time function (V-m). Two earth models are used: the average ocean model of Regan and Anderson [1984] (R-A) and PREM [Dziewonski and Anderson, 1981]. (b) Error versus centroid-depth curves for the Sumba event for the C-m, V-m, and uniform-slip fault (C-f) models.
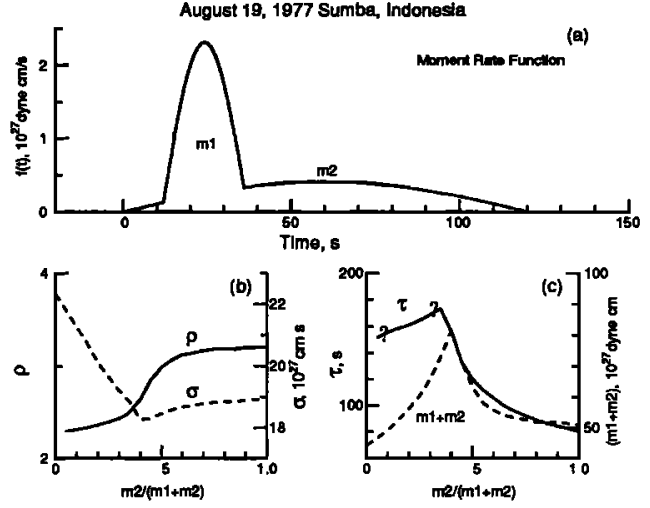

Fig. 3. The Sumba earthquake results. (a) The moment rate function. (b) The errors, $\rho$ (solid line) and $\sigma$ (256-s period) (dashed line), versus moment ratio curves. (c) The process time, $\tau$, (solid line) and total seismic moment, $m_{1}+m_{2}$, (dashed line) versus moment ratio curves.

tation, and propagation corrections, Consideration of the additional model variance will result in broader bounds [ZKK]

The source duration and centroid depth of the Tonga event have been estimated in several previous investigations, using either a boxcar for the time function [e.g., Lundgren and Okal, 1988; Giardini, 1984], or an $\omega^{2}$-model for the total moment [Silver and Jordan, 1983]. Our results are generally consistent with the previous studies, but we have better resolution of the broadband source function.

\section{August 19, 1977 Sumba, Indonesia earthquake}

For the Sumba earthquake (0608:55.2UT, $11.085^{\circ} \mathrm{S}, 118.464^{\circ} \mathrm{E}$, $m_{b}=7.0, M_{s}=7.9, M_{W}=8.5$ ), a preferred model of the moment-rate function (Figure 3a) is obtained, which comprises two components with a total process time of $120 \mathrm{~s}$. The 120-s process time is obtained by assuming that the first component with moment $m_{1}$, starting 12-s after the origin time, has a process time of $24 \mathrm{~s}$; and the second component, starting at $0 \mathrm{~s}$, has a moment $m_{2}$ equal to $m_{1}$. The assumptions on the start time and process time of the first component are based upon the body wave time function of the earthquake obtained by Lynnes and Lay [1988] and Kikuchi and Fukao [1987]. The size of the moment assigned to the second component, $50 \%$ of the total moment, is estimated by comparing errors obtained in inversions for various assumed moment ratios and by matching $m_{1}$ with the moment obtained from body waves for this earthquake. The assumption that the second component starts at the origin time is somewhat arbitrary; however, using different start times for the second component yields similar estimates of the depth and moment (with differences less than 5\%).

Figures $3 b$ and $3 c$ show errors, $\rho$ and $\sigma$, process times, and seismic moments obtained for various moment ratios, $m_{2} /\left(m_{1}+m_{2}\right)$. For a ratio of about $50 \%$, the process time is 120 $\mathrm{s}$, and the seismic moment is $63 \times 10^{20} \mathrm{~N} \mathrm{~m}$ (for $36-\mathrm{km}$ centroid depth); the first component has a moment almost the same as the $30 \times 10^{20} \mathrm{~N} \mathrm{~m}$ obtained from body waves [Lynnes and Lay, 1988]. For a ratio of $42 \%$, the seismic moment is $79 \times 10^{20} \mathrm{~N} \mathrm{~m}$ (for $31-\mathrm{km}$ centroid depth), and $m_{1}$ is 1.5 times as large as the moment obtained from body waves, so we have a weak preference for the $50 \%$ ratio case. For a smaller ratio (less than $40 \%$ ), the moment-rate function with any process time does not have sufficient phase delay to match the data; thus, $\sigma$ is large.

Using the V-m model, we obtained a $36-\mathrm{km}$ centroid depth, which is significantly shallower than the 44-km depth obtained for a $\mathrm{C}-\mathrm{m}$ model with an optimal 80-s duration determined using the method of $\mathrm{ZKa}$, and the $46-\mathrm{km}$ depth for a model of bilateral 
faulting with constant dislocation on the fault plane (C-f) obtained by $\mathrm{ZKa}$. The variance in the inversion using $\mathrm{V}-\mathrm{m}$ is slightly smaller than that using $\mathrm{C}-\mathrm{m}$ and $\mathrm{C}-\mathrm{f}$, with the minimum $\rho$ for $\mathrm{V}-\mathrm{m}$ reduced from that for $\mathrm{C}-\mathrm{m}$ and $\mathrm{C}-\mathrm{f}$ by less that $10 \%$ (Figure 2b). Given the small size of our data set, the small relative variance reduction and large depth variation obtained for different finiteness models indicate that determination of the centroid depth of the Sumba earthquake is difficult. The V-m model for the Tonga earthquake gave a factor of two reduction of $\rho$ relative to the $\mathrm{C}-\mathrm{m}$ model, which is possibly due to the higher quality data available for that study.

From the observations of fundamental spheroidal modes in the frequency interval $1-11 \mathrm{mHz}$ (50\% of the data have periods less than $155 \mathrm{~s}$ ) recorded by IDA stations, Silver et al. [1986] obtain $\tau_{c}=25 \mathrm{~s}$ and a depth less than $25 \mathrm{~km}$. Their $25-\mathrm{s} \tau_{c}$ corresponds to an 43-s process time (for a boxcar time function), consistent with that of ZKa using the same earth model; however, their estimate of depth appears much shallower than that obtained in this study. There are several possible causes of this discrepancy. First, because of the very large size of the event, all usable phases at IDA stations correspond to waves with very long propagation paths. Secondly, at periods shorter than $150 \mathrm{~s}$, the effect of the earth's lateral heterogeneity is severe, and presently available earth models do not give reliable path corrections. This effect is most pronounced for free oscillation analysis. Thirdly, the differences between depths obtained for various earth models are not negligible $[\mathrm{ZKb}]$. For these reasons, accurate transfer functions for a broad frequency range including excitation and effects of lateral heterogeneities on both amplitudes and phase are required for ultimate resolution of the discrepancy.

\section{Discussion and Conclusions}

Table 1 summarizes the source function results. For the Tonga earthquake, the use of the variable moment-rate function with 165 -s process time reduces the error $\rho$ in the moment tensor inversion significantly compared to assuming the boxcar time function with 84-s process time. For the Sumba earthquake, although the decrease in $\rho$ using the variable moment-rate compared with the C-m and C-f models is much smaller, the variable moment-rate is still preferred, since it approximately fits the body waves.

Relative to the solutions assuming boxcar time functions, the variable moment-rate function models have longer source process times and shorter apparent durations, but about the same centroid times for both the Tonga and Sumba earthquakes. This suggests that apparent duration and process time can be used to provide a first order characterization of the source complexity of large earthquake rupture process.

The seismic moments and total process times obtained in this long-period surface wave study differ from body waves estimates for these two great earthquakes. This suggests that much of the energy of the long-period waves is radiated from a larger area and over longer time scale than the energy in the body waves.

Table 1. Low Frequency Properties of two Great Earthquakes

\begin{tabular}{lcccc}
\hline \multicolumn{1}{c}{ Event } & Tonga 1977 & $\mathrm{M}_{\mathrm{w}}=8.2$ & Sumba 1977 & $\mathrm{M}_{\mathrm{w}}=8.5$ \\
Moment-Rate Model & $\mathrm{C}-\mathrm{m}^{*}$ & $\mathrm{~V}^{*} \mathrm{~m}^{* *}$ & $\mathrm{C}-\mathrm{m}$ & $\mathrm{V}-\mathrm{m}$ \\
\hline Apparent Duration (s) & 84 & 47 & 80 & $25-27$ \\
Total Process Time (s) & 84 & 165 & 80 & $120-145$ \\
Centroid Time (s) & 42 & 48 & 40 & $42-44$ \\
Centroid Depth (km) & 91 & 96 & 42 & $31-36$ \\
Moment $\dagger$ & 17 & 21.5 & 56 & $63-79$ \\
\hline *: Constant moment rate function (Boxcar) \\
**: Variable moment rate function \\
$\dagger:$ The seismic moment (in units of $10^{20} \mathrm{~N} \mathrm{~m}$ ) of the best double \\
couple of the moment tensor.
\end{tabular}

This interpretation supports the asperity model of rupture process of earthquakes.

Acknowledgments. We thank Paul G. Silver and the reviewers for helpful comments. This work was supported by NSF grant.

\section{References}

Beck, S. L., and L. J. Ruff, Rupture process of the great 1963 Kurile Islands earthquake sequence: Asperity interaction and multiple event rupture, $J$. Geophys. Res., 92, 14123-14138, 1987.

Christensen, D. H., and T. Lay, Large earthquakes in the Tonga region associated with subduction of the Louisville Ridge, $J$. Geophys. Res., 93, 13367-13389, 1988.

Dziewonski, A. M., and D. L. Anderson, Preliminary reference Earth model, Phys. Earth Planet. Inter., 25, 297-356, 1981.

Dziewonski, A. M., and J. M. Steim, Dispersion and attenuation of mantle waves through waveform inversion, Geophys. $J . R$. astr. Soc., 70, 503-527, 1982.

Dziewonski, A. M., and J. H. Woodhouse, An experiment in systematic study of global seismicity: Centroid-moment tensor solutions for 201 moderate and large earthquakes of $1981, J$. Geophys. Res., 88, 3247-3271, 1983.

Ekström, G., and A. M. Dziewonski, A very broad band analysis of the Michoacan, Mexico earthquake of September 19, 1985, Geophys. Res. Lett., 13, 605-608, 1986.

Gilbert, F., An introduction to low-frequency seismology, Proc. Enrico Fermi Int. Sch. Phys., 78, 40-81, 1980.

Hartzell, S. H., and T. H. Heaton, Teleseismic time functions for large, shallow subduction zone earthquakes, Bull. Seism. Soc. Am., 75, 965-1004, 1985.

Kanamori, H., and J. W. Given, Use of long-period surface waves for rapid determination of earthquake-source parameters, Phys. Earth Planet. Inter., 27, 8-31, 1981.

Kikuchi, M., and Y. Fukao, Inversion of long-period P-waves from great earthquakes along subduction zones, Tectonophysics, 144, 231-247, 1987.

Lynnes, C. S., and T. Lay, Source process of the great 1977 Sumba earthquake, J. Geophys. Res., 93, 13407-13420, 1988.

Regan, J., and D. L. Anderson, Anisotropic models of the upper mantle, Phys. Earth Planet. Inter., 35, 227-263, 1984.

Schwartz, S., and L. J. Ruff, The 1968 Tokachi-Oki and the 1969 Kurile Islands earthquakes: Variability in the rupture process, J. Geophys. Res., 90, 8613-8626, 1985.

Silver, P. G., and T. H. Jordan, Optimal estimation of scalar seismic moment. Geophys. J. R. astr. Soc., 70, 755-787, 1982.

Silver, P. G., and T. H. Jordan, Total moment spectra of fourteen large earthquakes, J. Geophys. Res., 88, 3273-3293, 1983.

Silver, P. G., M. A. Riedesel, T. H. Jordan, and A. F. Sheehan, Low frequency properties of the Sumbawa earthquake of 1977. EOS Trans. AGU, 67, 309, 1986.

Woodhouse, J. H., and A. M. Dziewonski, Mapping the upper mantle: Three-dimensional modeling of Earth structure by inversion of seismic waveforms, J. Geophys. Res., 89, 59535986, 1984.

Zhang, J., and H. Kanamori, Source finiteness of large earthquakes measured from long-period Rayleigh waves, $P$ hys. Earth Planet. Inter., 52, 56-84, 1988a.

Zhang, J., and H. Kanamori, Depths of large earthquakes determined from long-period Rayleigh waves, J. Geophys. Res., 93, 4850-4868, 1988b.

J. Zhang and T. Lay, Department of Geological Sciences, The University of Michigan, Ann Arbor, MI 48109.

(Received: December 7, 1988;

Revised: February 17, 1989;

Accepted: February 20, 1989) 